\title{
Co-contagion diffusion on multilayer networks
}

\author{
Ho-Chun Herbert Chang ${ }^{1,2,4^{*} \dagger}$ (D) and Feng Fu I,3† $^{2}$
}

\author{
${ }^{*}$ Correspondence: \\ herbert.hc.chang@gmail.com \\ ${ }^{\dagger}$ Ho-Chun Herbert Chang and Feng \\ Fu contributed equally to this work. \\ 'School of Informatics, University of \\ Edinburgh, Edinburgh, UK \\ ${ }^{2}$ Department of Mathematics, \\ Dartmouth College, 03755 Hanover, \\ USA \\ Full list of author information is \\ available at the end of the article
}

\begin{abstract}
This study examines the interface of three elements during co-contagion diffusion: the synergy between contagions, the dormancy rate of each individual contagion, and the multiplex network topology. Dormancy is defined as a weaker form of "immunity," where dormant nodes no longer actively participate in diffusion, but are still susceptible to infection. The proposed model extends the literature on threshold models, and demonstrates intricate interdependencies between different graph structures. Our simulations show that first, the faster contagion induces branching on the slower contagion; second, shorter characteristic path lengths diminish the impact of dormancy in lowering diffusion. Third, when two long-range graphs are paired, the faster contagion depends on both dormancy rates, whereas the slower contagion depends only on its own; fourth, synergistic contagions are less sensitive to dormancy, and have a wider window to diffuse. Furthermore, when long-range and spatially constrained graphs are paired, ring vaccination occurs on the spatial graph and produces partial diffusion, due to dormant, surrounding nodes. The spatial contagion depends on both dormancy rates whereas the long-range contagion depends on only its own.
\end{abstract}

Keywords: Complex contagions, Network diffusion, Stochastic modeling

\section{Introduction}

From misinformation to technological uptake, interconnected domains increasingly demand sophisticated models to understand their diffusion phenomenon. Under the framework of graphs and networks, contemporary research in social contagion diffusion follows three directions: an ecology of contagions, the mechanisms of diffusion, and population structures (Guilbeault et al. 2018). This paper addresses the first two, where we examine the simultaneous diffusion of two contagions constraint to different layer pairs in multiplex networks. Specifically, we study the interaction of synergy and dormancy.

Synergy denotes the phenomenon when two contagions spread faster together, and the level of synergy describes the speed-up associated. Contagions are also typically associated with a dormancy factor, where they no longer actively spread the contagion after a certain period of time. At a glance, synergy and dormancy appear to be naturally opposing forces. However, their interaction is not as simple as additive cancellation. For instance, synergistic contagions should diffuse faster together, since greater density implies greater adoption probability. However, if one contagion diffuses much faster than the others, it

(c) The Author(s). 2019 Open Access This article is distributed under the terms of the Creative Commons Attribution 4.0 International License (http://creativecommons.org/licenses/by/4.0/), which permits unrestricted use, distribution, and reproduction in any medium, provided you give appropriate credit to the original author(s) and the source, provide a link to the Creative Commons license, and indicate if changes were made. 
may introduce dormancy within the population, thus "vaccinating" the population against subsequent contagion and produces percolation.

Prior results show probabilistic branching and percolation based on synergy and dormancy ratios (Chang and $\mathrm{Fu} 2018$ ). While insightful from a complex network perspective, prior analysis only encapsulates lattice and regular-random-graphs, and thus limits direct application to today's real systems. This paper aims to bridge that gap- we generalize diffusion behavior based on common graph structures, and demonstrate case-specific phenomenon based on network properties such as shortest path, group boundaries, and degree distribution.

Co-infection is a notion in epidemiology that describes multiple contagions interfering with each other, and can simultaneously infect a host (Nowak and May 1994). Thus, co-infection can be extended to model social contagions as, like infectious diseases, behaviors do not spread in isolation.

In the domain of epidemiology, Cai et al. have proposed a co-evolutionary spreading model whose dynamics depend on the SIR model (Cai et al. 2015). They determine the conditions that induce phase transitions on nodes belonging to a giant component. Grassberger et al. further gives a thorough review of network topology influencing phase transitions (Grassberger et al. 2016). They note the importance of long-range dependencies on producing discontinuous phase transitions. Hebert-Dufresne and Althouse show on clustered networks, synergistic diffusion behaves differently than equivalent random networks. This suggests that clustering bolsters, rather than percolates, diffusion in comparing the synergistic and single contagion case (Hébert-Dufresne and Althouse 2015). These studies have been largely constrained to single layer networks. For the case of multiplex networks, Azimi-Tafreshi used general percolation theory to compute the fraction of nodes that are infected at equilibrium. The author describes multiplex networks using joint-degree distribution, then, given the overlapping edges, computes the final state and shows the emergence of a tri-critical point (Azimi-Tafreshi 2016). Synergy in this field has been defined many ways, for instance, dynamically inferred from the neighborhood of susceptible-infected pairs (Pérez-Reche et al. 2011).

While co-diffusion has its roots in epidemiology, contemporary systems benefit greatly from contagion interaction models. Multilayer models can also be better understood in the context of infrastructural, institutional, or functional divisions. Examples include the internet and power grids (Buldyrev et al. 2010), transportation systems (Gu et al. 2011), and information on different social media platforms (Buono et al. 2014). Models have been developed to encapsulate a fraction of all nodes, with respect to real networks.

For social contagions, prior research has been divided between successive and simultaneous contagions (Wang et al. 2019); this research focuses on the latter. A common paradigm has been through evolutionary games, which not only provides a vocabulary for characterizing cooperation versus competition, but generalizes well across different mechanisms and domains (Zinoviev and Duong 2011; Qiu et al. 2012). For instance, Jiang (Jiang et al. 2014) models information diffusion as a game on social networks, where mutations are interpreted as new information. Teaching activity and information sharing has been modeled similarly by Szolnoki (Szolnoki and Perc 2008; 2013), with concentrated efforts being directed towards multilayer networks (Jiang et al. 2014; Wang et al. 2015; Perc et al. 2013). In particular, research has shown the importance of topological features, such as collective influence by degree (Szolnoki and Perc 2016), stochasticity 
and noise (Perc and Marhl 2006), and strategy/topology coevolution (Wang et al. 2014). Shu et al. (2017) study contagions on two interdependent lattices, which are spatially constrained.

More closely related to our work are complex contagion threshold models. Zarazade et al. have discovered diffusion synergy between correlated platforms, such as Youtube and Google Play when a new album is released (Zarazade et al. 2017). On the other hand, they note URL sharing is competitive. The focus of theoretical models has been diverse, ranging from a pair of simple and complex layers (Czaplicka et al. 2016), to trusted and distrusted edges between layers (Srivastava et al. 2016). These studies focus on competing contagions, though recently there has been directed research effort toward synergy. Liu et al. consider the diffusion of two contagions constrained to two layers (Liu et al. 2018; 2018), considering diffusion density of other contagions as synergy. Chang and Fu build on this prior model by quantifying the different types of synergy using a formulation similar to Loewe Additivity, and introduce the effects of dormancy on diffusion behavior (Chang and Fu 2018).

\section{Model and methods}

The purpose of this paper is to understand the general properties of multilayer diffusion for network layers of fixed degree, and show the intricate relationship between synergy, dormancy, and topology. This requires a model that describes synergistic diffusion, as well as a way to parameterize dormancy. Investigations on topology arise from different multilayer graph pairings, for instance, a lattice graph paired with a random graph. As a point of clarification, our study is conducted on edge-colored multiplex networks. These are networks where the set of nodes across networks are the same, but not the edges. In other words, the neighborhoods for each node on different layers is different.

\section{Co-contagion diffusion process}

Suppose we have two contagions, Contagion $A$ and Contagion $B$. Associated with the two contagions are two network layers who share nodes, but not edges - an edge-colored multiplex network as shown in Fig. 1. Contagion $A$ spreads on one layer, and Contagion $B$ the other. Using the framework of threshold models, each node is assigned a random threshold between $[0,1]$, and adopts a contagion when some function of its neighbors exceeds this threshold.

Each node on can attain four possible states: not infected/naïve (Ø), $A, B$, and $A B$. Respectively, these denote no infection, infected by Contagion $A$, infected by Contagion $B$ and infected by both (co-infection). Additionally, a node is either active or dormant, represented by a binary variable, 1 denoting it is active and 0 denoting it is not. Thus, each node $i$ is represented by the tuple (State, Active).

Figure 1 illustrates the process of diffusion, with a lattice layer on the top and a powerlaw network on the bottom. Here, as with any edge-colored multilayer network, nodes have a one-to-one correspondence to themselves in both layers. Starting out with a node that has adapted both Contagion $A$ and Contagion $B$, shown with the blue node. Contagion $A$ only diffuses on the top lattice layer, and Contagion $B$ diffuses on the bottom power-law layer. Yellow nodes denote nodes that are susceptible to infection, whose adaption probability depends on neighbors who are infected (blue over total neighbors). On 


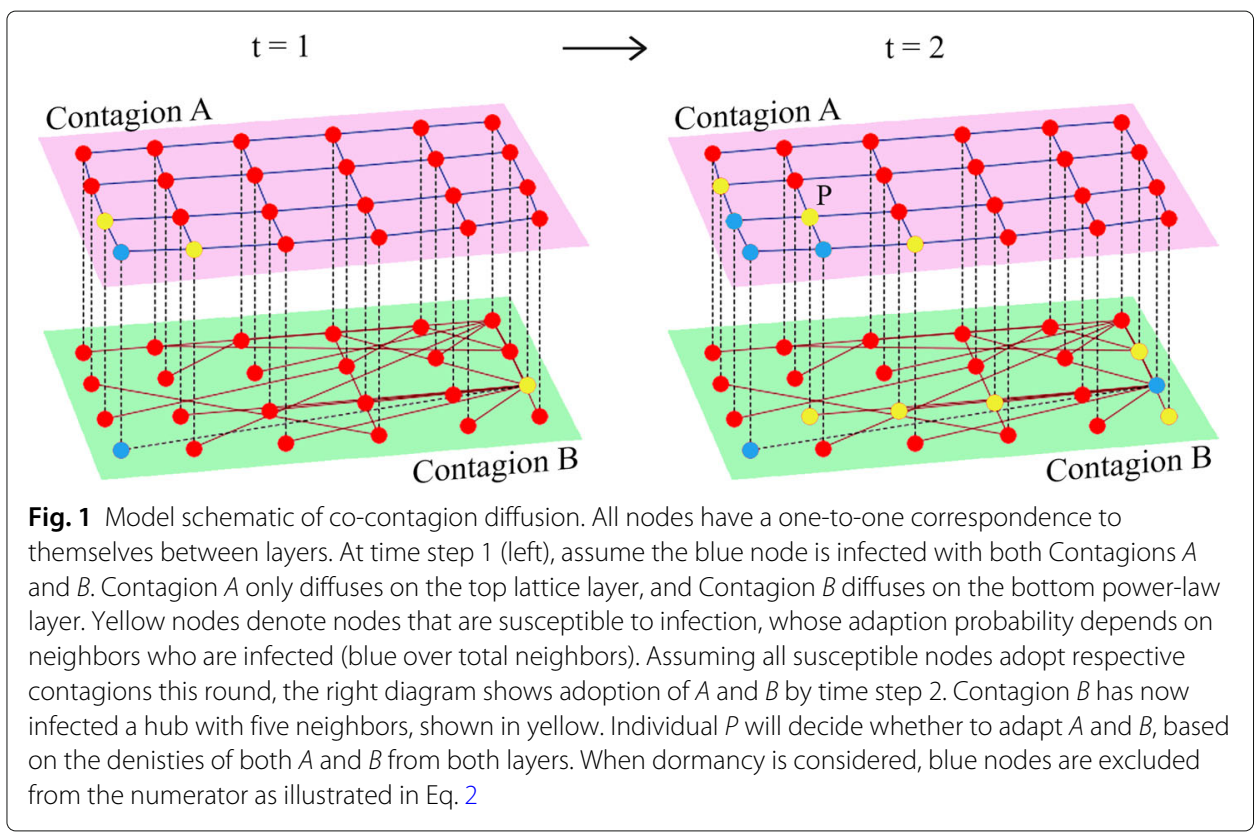

the top layer, the node has four (spatial) neighbors, where as in the bottom layer it has one long-range neighbor, shown with a dotted line.

For the sake of illustration, suppose all susceptible nodes adopt their contagions. Then at time step 2, Contagion $B$ will have infected a hub, producing five susceptible neighbors. By the next round, individual $P$ will have decided whether to adopt $A$ or $B$, based on the densities from both layers. Note, although hubs spread a contagion quickly, they are also difficult to infect based on their density function- hence, the diffusion on the other layer can positively boost a node's adoption probability. When dormancy is considered, blue nodes are discounted from the numerator thus diminishing the density.

In other words, while there is no interlayer contagion since the diffusion of each contagion is constrained to its own network layer, the "adoption" probabilities depend on both contagions. This creates interference based on the graph structures, synergy and dormancy. A broad overview of the schematic is given in Fig. 2.

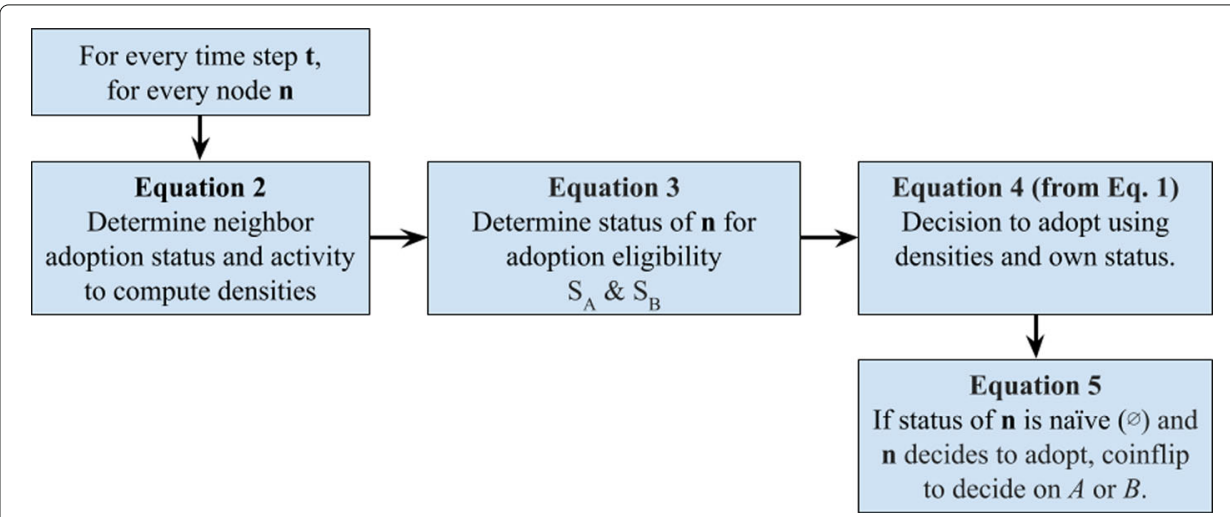

Fig. 2 Algorithmic flowchart of simulating the co-contagion diffusion process 
Specifically, the probability of diffusion is described by the multivariate Hill function (Chang and $\mathrm{Fu} 2018$ ), which governs canonical logistic growth. The concavity, controlled by parameter $\alpha$, determines whether the additivity between contagions is synergistic or antagonistic. Equation 1 gives the general form of the adoption function.

$$
P(i \leftarrow A \text { or } B)=\frac{\left(1-S_{A}(i)\right)\left(\frac{[A]}{K_{A}}\right)^{\alpha}+\left(1-S_{B}(i)\right)\left(\frac{[B]}{K_{B}}\right)^{\alpha}}{1+\left(1-S_{A}(i)\right)\left(\frac{[A]}{K_{A}}\right)^{\alpha}+\left(1-S_{B}(i)\right)\left(\frac{[B]}{K_{B}}\right)^{\alpha}}
$$

with the density described as:

$$
[A]=\frac{\text { Active neighbors inf. } \mathrm{w} / \mathrm{A}}{\text { Total Neighbors }} \quad[B]=\frac{\text { Active neighbors inf. w/ B }}{\text { Total Neighbors }}
$$

The left arrow denotes node $i$ adopting $A$ or $B$. The $K_{j}$ 's denote the attractiveness of a contagion $j$, and reflects the canonical linear threshold model if set to 1 . The indicator functions notes the status of a node, then reduces the diffusion probability to uni-variate sub-cases for logistic diffusion. Explicitly:

$$
S_{A}(i)=\left\{\begin{array}{ll}
1 & \text { if } i \text { adopts A } \\
0 & \text { if otherwise }
\end{array} \quad S_{B}(i)= \begin{cases}1 & \text { if } i \text { adopts B } \\
0 & \text { if otherwise }\end{cases}\right.
$$

For instance, if node $i$ has adopted Contagion $A$, then $S_{A}(i)=1$ such that the terms containing Contagion $A$ drop away.

$$
P\left(i \leftarrow A \text { or } B \mid S_{A}=1, S_{B}=0\right)=P(i \leftarrow B)=\frac{\left(\frac{[B]}{K_{B}}\right)^{\alpha}}{1+\left(\frac{[B]}{K_{B}}\right)^{\alpha}}
$$

However, if the state of node $i$ is uninfected (Ø), then it can take on one of $A$ and $B$. Equation 1 only denotes a binary decision whether to adopt or not. The choice between the two is settled by a coin-toss, weighed by their relative densities in Eq. 5 .

$$
\begin{aligned}
& P\left(i \leftarrow A \mid S_{A}=0, S_{B}=0\right)=\frac{\left(\frac{[A]}{K_{A}}\right)^{\alpha}}{\left(\frac{[A]}{K_{A}}\right)^{\alpha}+\left(\frac{[B]}{K_{B}}\right)^{\alpha}} \\
& P\left(i \leftarrow B \mid S_{A}=0, S_{B}=0\right)=\frac{\left(\frac{[B]}{K_{B}}\right)^{\alpha}}{\left(\frac{[A]}{K_{A}}\right)^{\alpha}+\left(\frac{[B]}{K_{B}}\right)^{\alpha}}
\end{aligned}
$$

This model draws influence from pharmacology (Foucquier and Guedj 2015), where researchers consider the efficacy of drugs when they are used in conjunction. Drugs interact synergistically if they yield better results together, or antagonistically if reduced efficacy is observed. As mentioned prior, $\alpha$ is a critical parameter that controls for the synergy between contagions. This model of density-dependent performance can be extended naturally to the diffusion of complex contagions (Centola 2018).

\section{Dormancy $\tau$}

Additionally, contagion $A$ and $B$ is associated with a dormancy constant $\tau_{A}$ and $\tau_{B}$ respectively. At a given time step, $\tau_{A}$ denotes the probability a node will go dormant and no longer actively diffuse the contagion. Dormant nodes are thus removed from the counts for density. Another way to interpret $\tau_{A}$ is as the frequency of going dormant; that is, at a given time step, $\tau_{A}$ percent of the population infected with $A$ will go dormant. 


\section{Parallel optimization}

To optimize the simulation algorithm, a few steps to parallelize the experimentation can be made since updates are synchronous. Node neighbors are stored in memory, and in addition to the graphs, we maintain three state vectors that record the following: nodes infected with $A$, nodes infected with $B$, and nodes that are active. We use two change vectors also of dimension equal to the number of nodes, denoted $\vec{\Delta}$ and $\vec{\gamma} \cdot \vec{\Delta} a$ denotes whether a node will change state based on Eq. 1 , and $\vec{\gamma}$ is the choice of $A$ over $B$ given in Eq. 5 . Then the status update rule for time step $t+1$ can be written as follows, in Eq. 6 .

$$
\vec{S}_{A, t+1}=\vec{S}_{A, t}+\vec{\Delta}\left(1-\vec{S}_{A, t}\right) \vec{\gamma} \quad \vec{S}_{B, t+1}=\vec{S}_{B, t}+\vec{\Delta}\left(1-\vec{S}_{B, t}\right)(1-\vec{\gamma})
$$

This tends to perform better when memory is not the primary constraint, but can lead to computational redundancies without heuristics.

\section{Simulation setup: graph parameters and topological properties}

Simulations were implemented using networkx (Hagberg et al. 2013). The pairwise multiplex networks include the following layers: perdiodic lattices (LAT), regularrandom-graphs (RRG), Erdős-Rényi random graphs (ERG), power-law graphs (PLG), and Watts-Strogatz graphs (WSG). Individual layers were generated using algorithms referenced in Table 1. To construct the edge-colored multilayer graph, nodes were then paired randomly one-to-one with nodes from another layer. Table 1 also shows the parameters used to initialize the graphs, for which pairings were made.

Updates were performed synchronously - for each time step, all nodes make an adaption decision using Eq. 1. This contrasts with asynchronous updates, where a single node is randomly chosen to make an adoption decision.

For all graph types, we fixed the total number of nodes to 6400, then graphs parameters were computed such that the average degree was four. This value is specified for regular-random-graphs and lattices, then computed explicitly for other graph types. The attractiveness parameters in Eq. 1 were set to be equal with $K_{A}=K_{B}=1.34$ as a hyper-parameter for analysis, and the synergy $\alpha$ was ranged from 0.5 to 5.0.

We describe each type of graphs in brief, noting key topological properties relevant in our analysis. Lattice graphs are graphs were each node has degree $k$ and forms a regular tiling with periodic boundary conditions. For instance, a square lattice has degree four, and serves as consistent baseline graph-type for spatial diffusion. A k-regular random graph is a graph where each node has degree $k$, making the distribution uniform (Steger and Wormald 1999). Unlike the lattice, it does not include tiling and has "long-range" connections. Erdős-Rényi random graphs are generated with two parameters $n$ and $p$, where $n$ is the number of nodes and $p$ is the probability an edge exists between any pair of two vertices (Erdős and Rényi 1960). Thus, the expected degree of a node is $(n-1) *$

Table 1 Initialization parameters for various models

\begin{tabular}{ll}
\hline Graph Type & Parameters \\
\hline Regular Random Graph (RRG) (Steger and Wormald 1999) & Degree $=4$ \\
Erdős-Rényi Graph (ERG) (Erdős and Rényi 1960) & Edges $=12800$ \\
Power-law Graph (PLG) (Holme and Kim 2002) & Random Edge per Node $=2$ \\
Lattice Graph (LAT) & Degree $=4$, Periodic $=$ True \\
Watts-Strogatz Small World (WSG) (Watts and Strogatz 1998) & Nodes $=6400, K=4, \beta=0.001$ \\
\hline
\end{tabular}


$p$. For the degree of four in our experiments, we found the value of $p$ to be $\frac{4}{6400-1}=$ $6.1251 * 10^{-4}$. Note, it is possible to generate a random graph by specifying the number of edges $m$ directly, which the algorithm does. Power-law graphs have power-law degree distributions; that is, the probability a node has degree $k$ is proportional to $\frac{1}{k^{\gamma}}$, with $\gamma>0$ (Holme and Kim 2002). These algorithms rely on preferential attachment- graphs are grown by adding new nodes, who connect to existing nodes proportional to their degree. The average degree was numerically verified to be 4 .

Small world graphs are typically characterized by high clustering coefficient and small characteristic path length, which denotes the typical separation of two vertices (Watts and Strogatz 1998). The Watts-Strogatz model is generated with three parameters: the number of nodes $n$, the degree $k$, and the rewiring probability $\beta$. The algorithm works as follows. First, $n$ nodes are arranged in a ring. Then each node is connected to $\frac{k}{2}$ neighbors on its right and $\frac{k}{2}$ to its left. For each edge, there is $\beta$ probability that the edge is rewired randomly, thus creating a long-range connection. Increasing $\beta$ gradually allows us to investigate the influence of shortest path on diffusion depth and diffusion rate. Having shorter paths on a network corresponds to a smaller characteristic path lengths, defined as the shortest path length for any pair of nodes. Hence, the Watts-Strogatz Model is a useful way to vary the shortest path (Fig. 3).

\section{Results}

\section{Shortest path and primacy jointly determine the impact of dormancy}

To investigate the phenomenon of branched diffusion, we consider the dynamics of primacy. We are interested in how the dormancy of the faster contagion affects the subsequent contagions. Watts-Strogatz Graphs are useful in parametrizing graphs of shortest path through adjustment of the rewiring probability $\beta$, as increasing the rewiring probability decreases the average shortest path, and enables us to investigate the influence of long-range dependencies in the diffusion. In this experiment, the multiplex network consists of two WSG layers- one for Contagion $A$ and one for Contagion $B$. We fix the rewiring probability of Contagion $A\left(\beta_{A}\right)$ to 0.01 , then vary $\beta_{B}$ from between $\frac{1}{800}$ to $\frac{1}{5}$.

Figure 3 shows that as the rewiring probability for $B$ increases, the faster the contagion diffuses due to the smaller shortest path. As $\beta_{B}$ increases, Contagion $B$ (blue) overtakes Contagion $A$ (red) in diffusion speed. Next, in Fig. 4, we introduce dormancy to investigate the effects of primacy in branch induction.

When $\beta_{B}$ is low as in Fig. 4a), Contagion $B$ diffuses more slowly than $A$. However, as $\beta_{B}$ increases in $4 \mathrm{~b}$ ), comparing the two thicker red lines demonstrates that its final depth

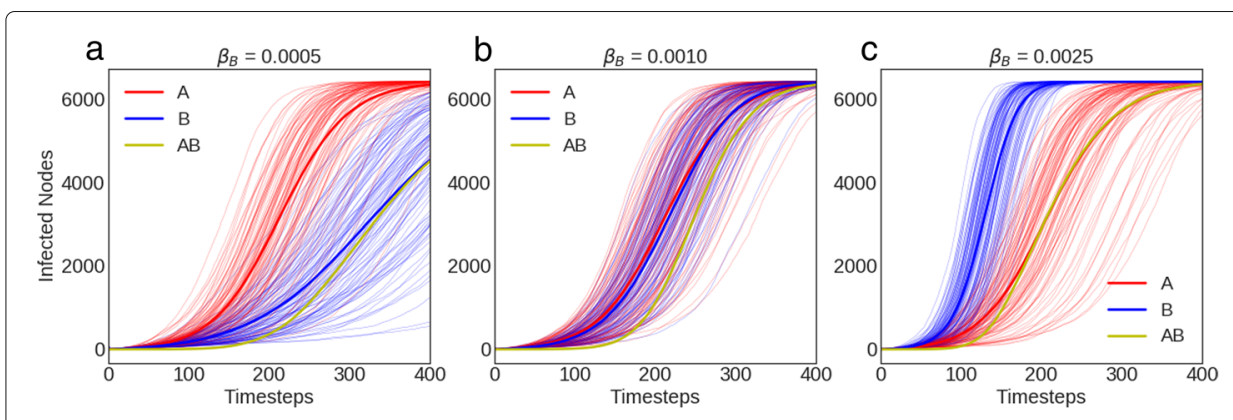

Fig. 3 Fixing $\beta_{A}$ to 0.01 , we increase $\beta_{B}$. When $\beta_{B}=0.005<\beta_{A}$, diffusion is slower (a). When $\beta_{B}=\beta_{A}$ they diffuse at equal rate $(\mathbf{b})$. When $\beta_{B}=0.05>\beta_{A}$, contagion $B$ is faster $(\mathbf{c})$. Here, $\tau_{A}=\tau_{B}=0.0, \alpha=1.0$ 

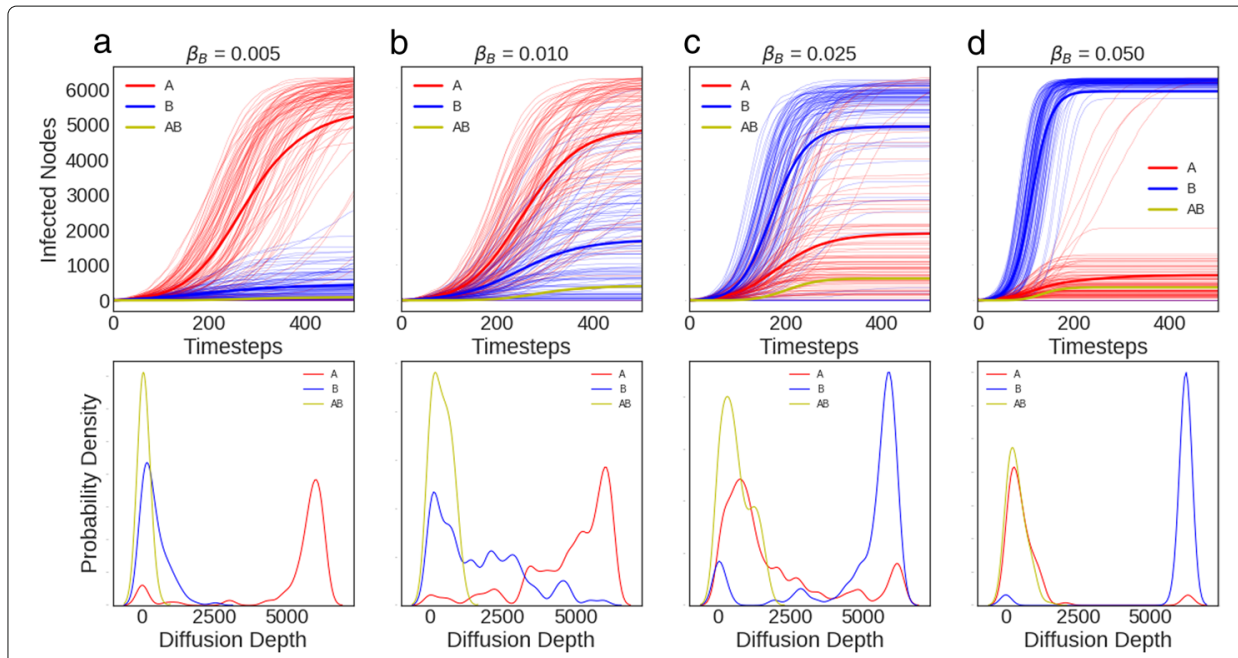

Fig. 4 Increasing $\beta_{B}$ with $\tau_{A}=0$ and a low $\tau_{B}=0.02$. When $\beta_{B}$ is low $(\mathbf{a})$, Contagion $B$ diffuses more slowly than $A$. However, as $\beta_{B}$ increases in $(\mathbf{b})$, even when Contagion $A$ still diffuses faster its diffusion depth has lowered. When $\beta_{B}>\beta_{A}(\mathbf{c})$, then Contagion $B$ diffuses more rapidly and lowers the diffusion ceiling of Contagion $A$. When $\beta_{B}$ is high (d), Contagion $A$ barely diffuses. $\alpha$ was fixed to 1.0

has lowered, even though Contagion $A$ still diffuses faster on average. This indicates a smaller proportion of trials that diffuse fully. When $\beta_{B}>\beta_{A}$ then Contagion $B$ diffuses more rapidly and hence lowers the diffusion ceiling of Contagion $A$. When the rewiring probability is low, it is easy to become blocked "spatially," similar to prior observations on lattices (Chang and $\mathrm{Fu}$ 2018). As we will discuss in Section 4, this is consistent with the observation that spatial aggregation enables ring vaccination.

The heatmaps in Fig. 5 show the phase transitions induced by $B$ on $A$, as we vary $\tau_{B}$ and $\beta_{B}$. We observe if $\tau_{B}=0$, then Contagion $A$ diffuses fully and uniformly, since even if Contagion $B$ diffuses faster, it does not introduce dormancy. However, when $\beta_{B}>\beta_{A}$, even low levels of $\tau_{B}$ induces percolation on Contagion $A$. This marks a phase transition. At $\tau_{B}=0.10$, the standard deviation is maximal, which indicates the point for which it is more likely $A$ transitions from the upper branch to the lower branch. For Contagion $B$, there is a linear relationship between the density of long-range relations and dormancy in determining diffusion depth. Note, as a property of the rewiring probability, as $\beta$ approaches 1 , more long-range connections are made and the graph approaches

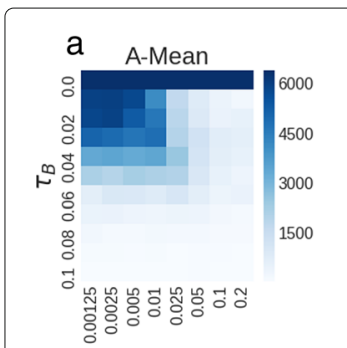

$\beta_{B}$

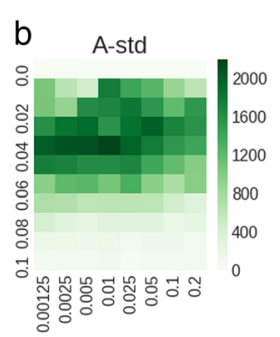

$\beta_{B}$

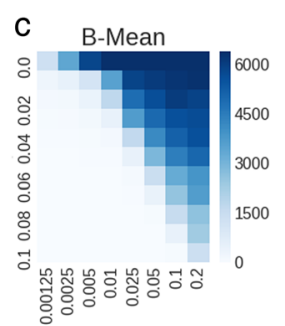

$\beta_{B}$

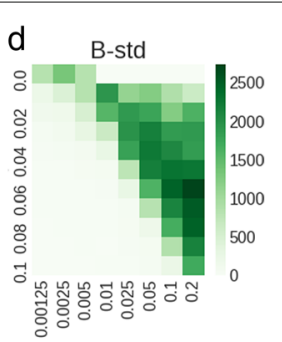

$\beta_{B}$

Fig. 5 Heatmaps of diffusion depth and variance for Contagation $A$ and Contagion $B$. Contagions diffuse on small-world graphs. The x-axes denote $\beta_{B}$, the rewiring probability of Contagion $B^{\prime}$ s layer, and the $y$-axes $\tau_{B}$, the dormancy rate of $B$. Panels $(\mathbf{a})$ and $(\mathbf{b})$ show the mean and standard deviation of Contagion $A^{\prime}$ s diffusion depth. A strong phase transition occurs when $\tau_{B}$ is non-zero at $\tau_{B}=\tau_{A}$. We fix $\beta_{A}=0.001, \tau_{A}=0$ and $\alpha=1$.0. An inverse relationship between $\tau_{B}$ and $\beta_{B}$ exists $(\mathbf{c})$ where the greatest instability occurs around $\tau_{B}=0.05$ and $\beta_{B}=0.20$ 
a random graph. Thus, our next step is to analyze diferent pairings of canonical graph structures, starting with long-long multiplex graphs.

\section{Degree distribution influences long-Long diffusion dynamics}

Having established the effects of shortest path and primacy, we compare the diffusion behavior between long-range graph pairing: RRG-ERG, ERG-PLG, and RRG-PLG. By long-long graph pairings, we refer to the existence of predominantly long-range connections on each layer. In contrast, lattice graphs only have short-range connections. These long-ranged graphs have comparable characteristic path length, and differ mostly in degree distribution- RRG degrees are uniform, ERGs are Poisson distributed, and PLGs by the power law. In order, these distributions increase in variance and "skewness". As a matter of terminology, we will refer to Contagions $A$ and Contagions $B$ by their associated layer (such as "the ERG Contagion") to avoid confusion.

With no dormancy on both layers, we observe the following order in diffusion speed: RRG > ERG > PLG. This is from directly observing this ordering across all timeseries, as shown in Fig. 6.

Given this ordering, we now investigate what produces the branching effect on longlong combinations. Figure 7 shows the diffusion averages of each of the graph pairings. The left heat map column shows the faster contagion all things equal, the right the slower contagion. Results from Chang and $\mathrm{Fu}$ (2018) suggest that branching usually occurs when the faster contagion has high dormancy and the slower one low. Setting $\tau_{A}=0.14$ and $\tau_{B}=0.02$, we show bi-modal diffusion curves and kernel density estimates of their branch values on the very right.

It is evident that the faster contagion depends on both $T_{A}$ and $T_{B}$ (first column), as shown by the diagonal line, whereas the subsequent contagion is much more sensitive to its own dormancy rate, on the y-axis. The rightmost column shows diffusion outcomes. Because of the existence of long-range connections, tri-modal diffusion does not occur.

Diffusion on these three graphs suggest the influence of the variance and skew of degree distribution. Given both the ERG and PLG exhibit right-skew in their degree distribution, this means a higher probability that a node with lower degree distribution will be sampled, on average, than on the uniformly distributed RRG. Therefore, ERG > PLG on the diffusion speed is a necessary conclusion as well.

We also confirm similar results on networks of other sizes, to control for finite size effect. Figure 8 shows branch induction is size invariant across RRG-ERG networks of size
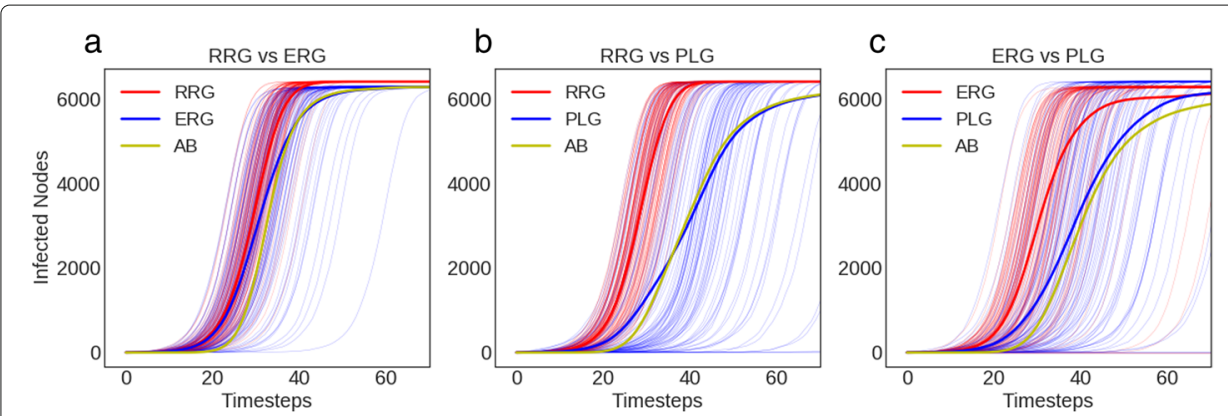

Fig. 6 Without dormancy and given random seed sampling, RRG contagions diffuse the fastest, followed by ERG graphs then PLG graphs. Parameters: $\tau_{A}=\tau_{B}=0$, alph $a=3.0$, with graph parameters given in Table 1 


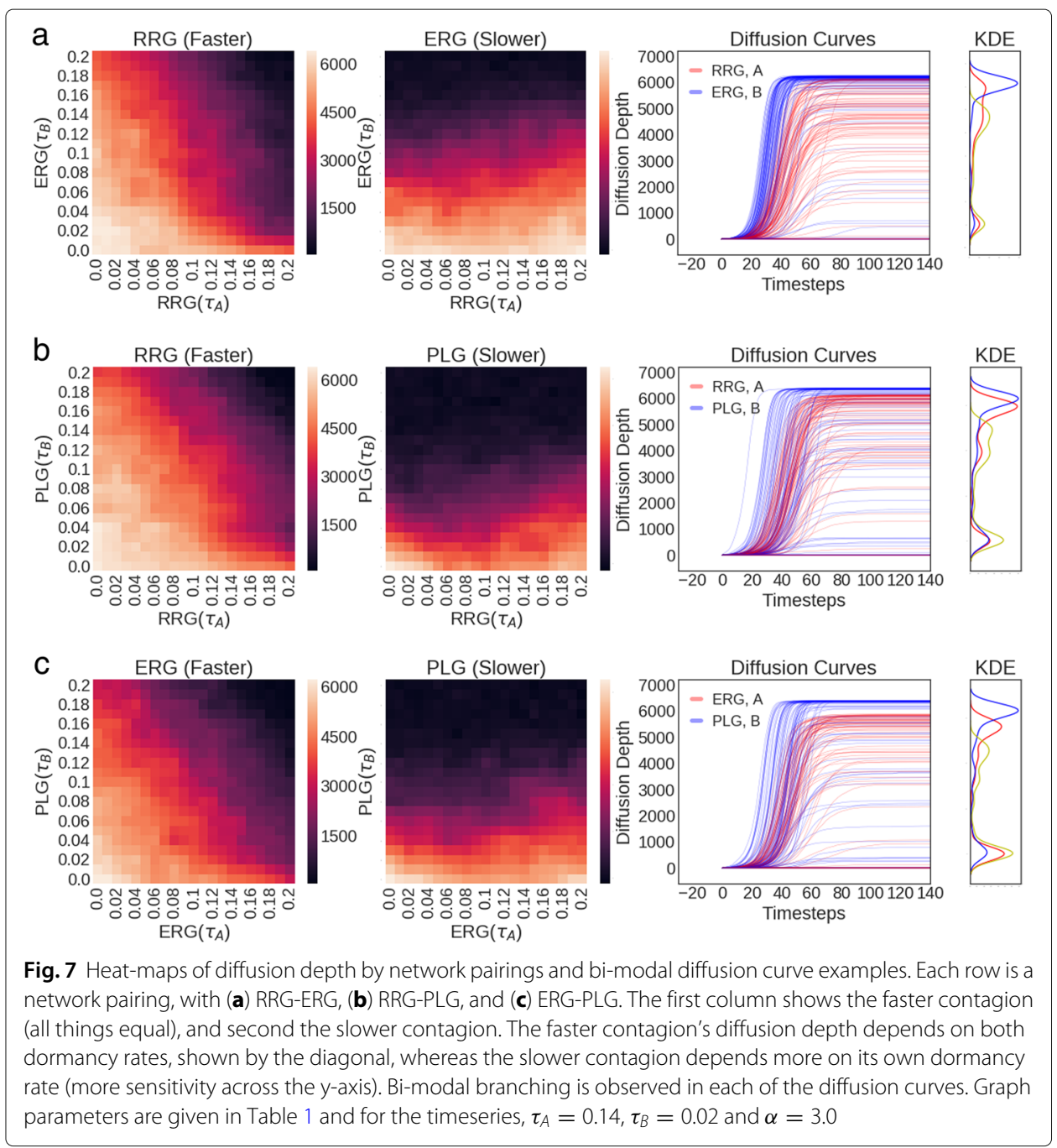

400, 1600 and 6400 . The time it takes for the slower contagion appears to be influenced by the size, although this requires further study.

\section{Synergy widens the diffusion window}

We observe the more synergistic contagions are, the faster both diffuse, which agrees with prior research (Chang and $\mathrm{Fu} 2018$ ). Additionally, as synergy increases ( $\alpha$ decreases), the resultant heat-maps grow less compressed. That is, the diffusion depth grows more sensitive to both $\tau_{A}$ and $\tau_{B}$ as $\alpha$ increases, and the lighter regions in the heatmap diminish. This implies the window for diffusion is wider for synergistic contagions.

To corroborate this point, we would expect that synergistic contagions perform better when their diffusion rates are comparable. Using an ERG-ERG pairing as a control, we compare the performances when one ERG layer has low dormancy $\left(\tau_{E R G, A}=0.02\right)$ and the other one is high $\tau_{B}=0.14$.

Figure 9 shows ERG-ERG, ERG-RRG and ERG-PLG pairings. Note, the thicker lines (blue, red, and yellow) denote the averages produced, which is not is not indicative of the actual diffusion outcome - a final depth of 3200 implies half the trials fully diffuse, while 


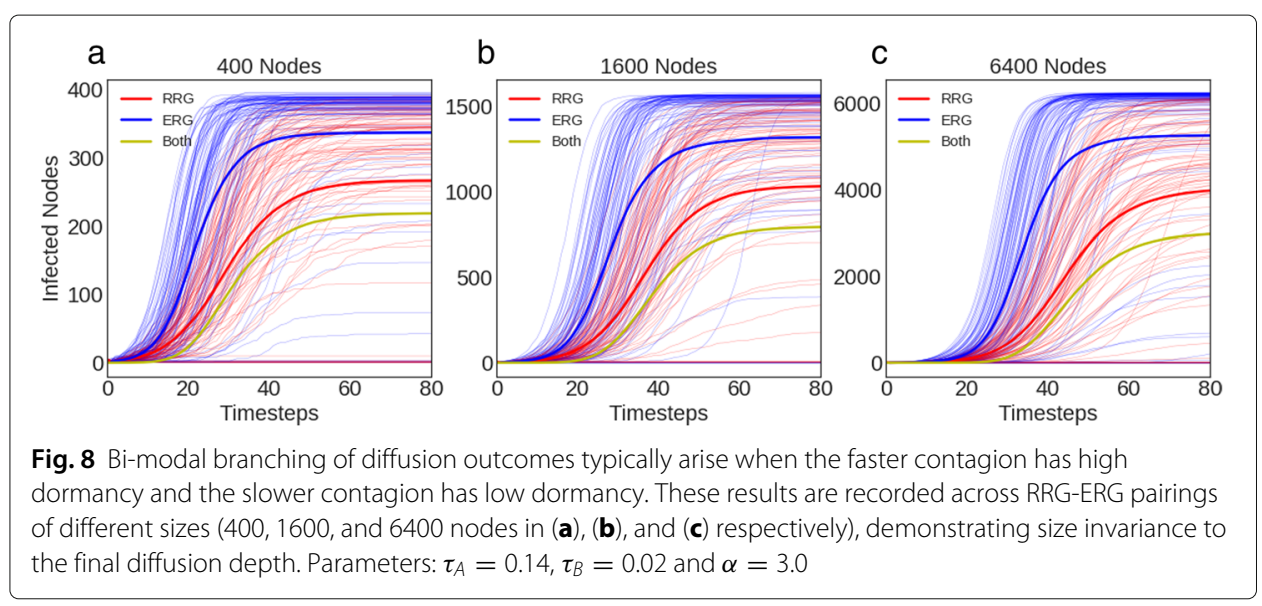

the rest barely take off. As expected, the ERG contagion with higher dormancy $\left(\tau_{B}=0.14\right)$ penetrates less deeply into the populace.

In contrast, on the ERG-RRG layer both diffuse fully more frequently, shown by the relatively higher red and blue lines, denoting a higher proportion of trials where both contagions diffuse fully. Since we have established RRG's are faster than ERG's all things held equal, when the relative diffusion rate of RRG's is lowered by $\tau=0.14$, the two diffusion rates become comparable. Synergy enhances diffusion particularly when diffusion rates are similar.

Since PLG's already diffuse slower than ERG's, the diffusion of the ERG layer with $\tau_{A}=0.02$ is comparable to the control (blue lines), since the PLG barely diffuses. These three cases demonstrate the sensitivity of diffusion outcome to the relative diffusion rate between contagions. Though not in the scope of this study, this result suggests that synergistic diffusion can be understood as a spectral analytic problem. This is supported by theoretical results for multilayer graphs, in which super diffusion has been shown using lifted Laplacians of the graphs and interlayer diffusion constants (Gomez et al. 2013).
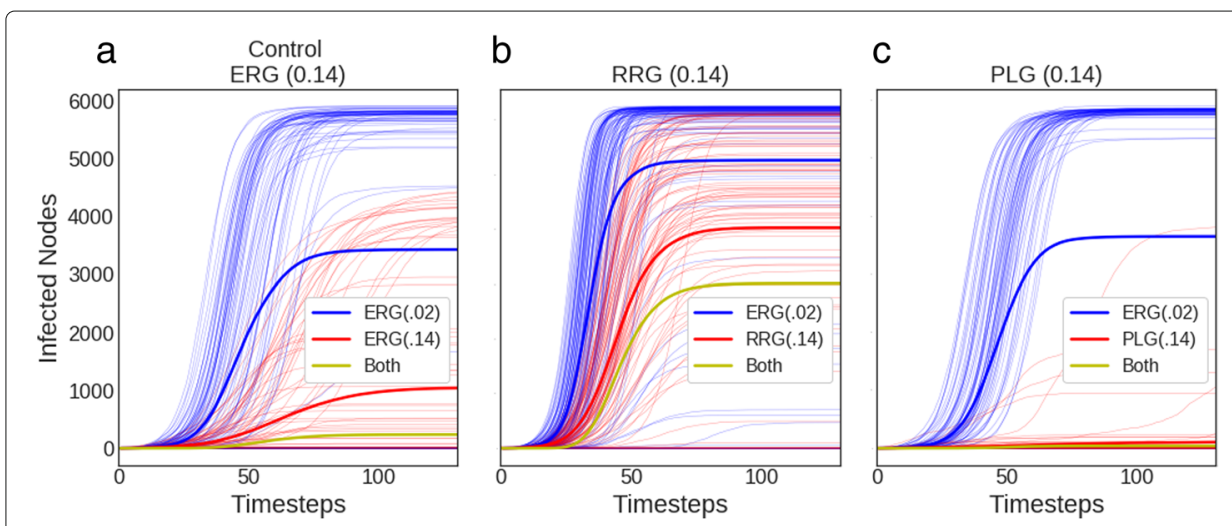

Fig. 9 In the ERG-ERG control $(\mathbf{a})$, we observe the blue branching $\left(\tau_{A}=0.02\right)$, with half the trials diffusing fully and the rest barely diffusing, evidenced by the thicker blue line near the middle. Because $\tau_{B}$ is 0.14 , the red line is much lower. In (b), a greater proportion of both contagions diffuse fully. Since the RRG layer diffuses faster all things equal, setting $\tau_{B}>\tau_{A}$ means the two contagions diffuse at a similar rate, indicating synergy inhances diffusion when their rates are similar. In (c), since PLG contagions already diffuse slower than ERG's, the ERG contagion penetrates similarly as the control, while the PLG barely diffuses. Here, $\alpha=3.0$ 
Thus, expressing dormancy as diffusion constants may yield insight into the timescales of diffusion.

\section{Spatial boundary enables ring vaccination in long-Short diffusion}

Lastly, we attempt to reproduce tri-modal branching. We define long-short pairings as a graph with predominantly long-range connections and one with predominantly shortrange connections, such as a lattice or a WSG with low rewiring probability. Prior studies have shown tri-modal branching occurring between the RRG-LAT multilayer graphs (Chang and $\mathrm{Fu}$ 2018). We show this is true on other long-short range combinations, specifically PLG-LAT and ERG-LAT pairings. Figure 10 shows the heat-maps of the longrange layer (first column) and the lattice layer (second column), then the diffusion curves on the right.

In contrast to the long-long-range diffusion heat maps, The long-range layer only depends on its own dormancy. This makes intuitive sense, as the spatially constrained lattice graph diffuses much slower than the PLG or ERG. On the contrary, the diffusion of the lattice layer depends heavily on the long-range layer. First, there is a steep transition between $\tau_{A}=0$ and $\tau_{A}=0.01$. Since the long-range contagion diffuses much faster, even low rates of dormancy induction produce a big drop in diffusion height. However, as dormancy rate increases for the long-range contagion, the long-range contagion slows and is eventually being overtaken by the lattice contagion. This can be observed when simultaneously viewing the heat maps - as the PLG turns dark vertically on the left, the LAT heat-map grows brighter above the long-range phase transition.

Note the strong diagonal line on the lattice heat map. This implies as long as $\tau_{L A T}<\tau_{A}$ for $A \in\{R R G, E R G, P L G\}$, then the lattice contagion will diffuse. Thus in general as $\tau_{A}$ increases, the deeper the lattice contagion diffuses.

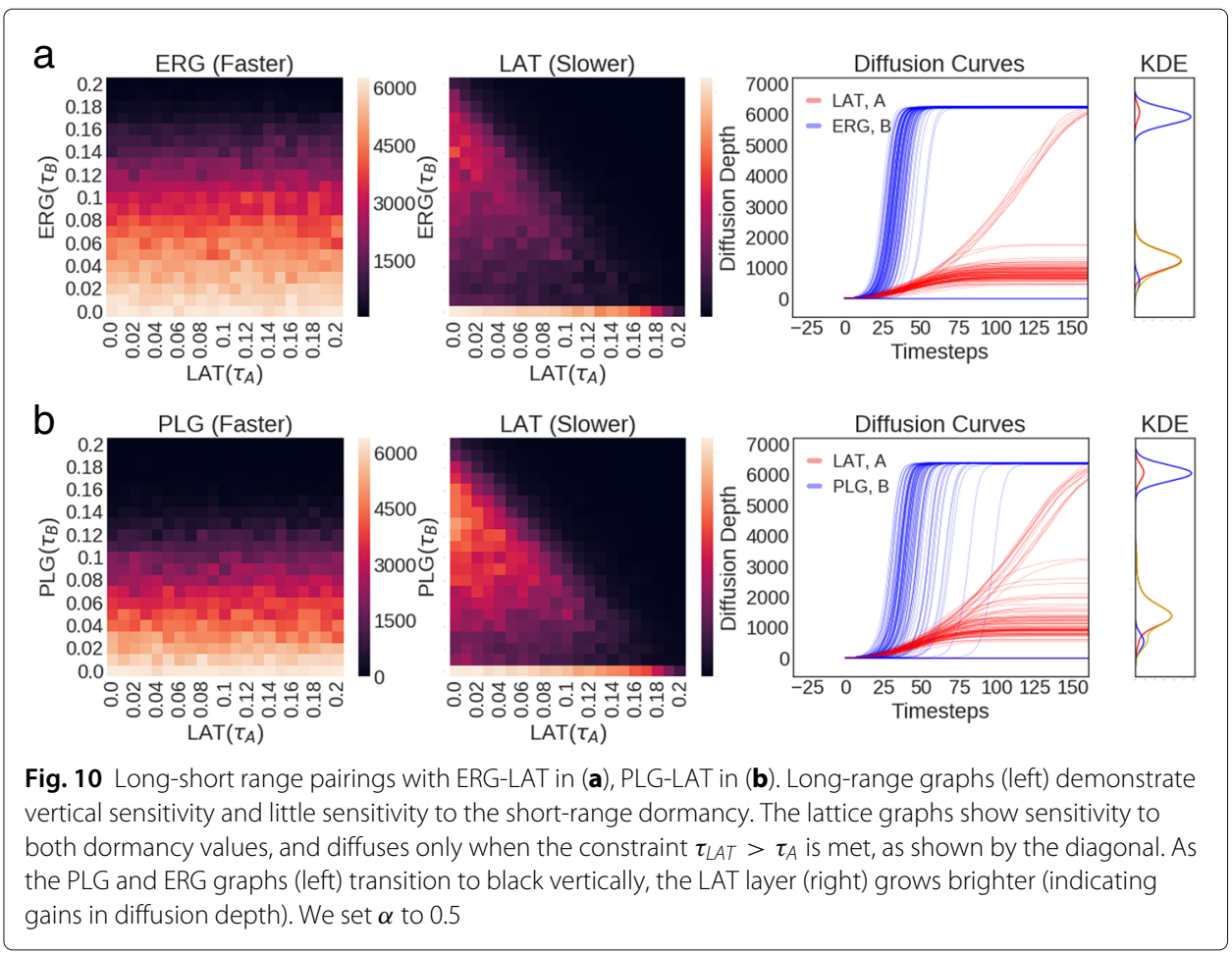




\section{Discussion and conclusion}

The purpose of this paper is to understand the general properties of multilayer diffusion for network layers of fixed degree, and results show the relationship between synergy, dormancy, and topology is very intricate. First, we established facts about diffusion primacy. The faster contagion, if containing non-zero dormancy, will induce branching on the slower contagion. We showed this by varying the rewiring probability of Watts-Strogatz graphs, and observed as the network shortest path decreases, the primacy relation between contagion changes and branch induction behavior switches between contagions. Second, synergistic contagions are found to have a more generous diffusion window - their diffusion depths are less sensitive to increases in dormancy compared to antagonistically additive contagions. Additionally, closer diffusion rates between two contagions yield more pronounced the synergistic effect.

Third, we investigate the interface of dormancy rates and topology. In long-range graph pairings, higher variance and right-skewness in degree distribution cause marginal, but important decreases in diffusion rate. The relative order in diffusion rate for long-range graphs is found to be regular-random graphs, ER-random graphs, and power law graphs. The faster contagion depends on both dormancy rates, and the slower one on its own. This relationship is flipped, however, when a long-range graph is paired with a spatially constrained lattice graph. The long-range graph depends mostly on its own dormancy rate, and increasing dormancy diminishes diffusion depth. For the lattice contagion, when the long-range dormancy is zero, then it only depends on its own dormancy rate. Its diffusion depth drops drastically when the long-range dormancy increases from 0 to a small value, but as the long-range dormancy continues to grow, the lattice contagion can diffuses deeper. The lattice contagion's diffusion rate is thus constrained by the faster contagion's dormancy from below, and from above by its own, as shown in Fig. 10.

Before extending this model to interpret real data, analysis of a few more properties is required. Real networks typically contain a subset of all nodes, so analysis of how these properties generalize to fractional coverage is required. Additionally, there have been promising empirical analyses recently. The influence of long-short range multiplex modeling has been suggested in the domain of ecology, with the super-diffusion of Trypansoma parasites (Stella et al. 2018). One observation was parasitic amplification owing to host-parasite and predator-prey interactions, which is related to our study of synergy. Similarly, synergistic diffusion may be analyzed in online social networks, by considering visibility and media relatedness (Jankowski et al. 2016) and topology (AlGaradi et al. 2016). Additionally, investigating the shift in sensitivity using lag-regression would yield quantitative insight regarding the precise relationship between dormancy and topology-specific diffusion rate, using techniques as described environmental epidemiology (Bhaskaran et al. 2013).

\section{Abbreviations \\ ERG: Erdős-Rényi random graph; LAT: Lattice; PLG: Power-law graph; RRG: regular-random-graph; WSG: Watts-Strogatz graph}

\section{Acknowledgements}

We'd like to thank the Discovery Cluster at Dartmouth College for computational resources. H.H.C. thanks the Dartmouth Senior Fellowship Graduate Fund and the University of Edinburgh, School of Informatics Masters Scholarship, and Lucas Pompe for coding suggestions. F.F. gratefully acknowledges the Dartmouth Faculty Startup Fund, the Neukom CompX Faculty Grant, Walter \& Constance Burke Research Initiation Award and NIH Roybal Center Pilot Grant. 
Authors' contributions

Both authors contributed equally to the manuscript.

\section{Funding}

Not applicable

Availability of data and materials

The datasets generated during the current study are available from the corresponding author on request.

\section{Competing interests}

The authors declare that they have no competing interests.

\section{Author details}

${ }^{1}$ School of Informatics, University of Edinburgh, Edinburgh, UK. ${ }^{2}$ Department of Mathematics, Dartmouth College, 03755 Hanover, USA. ${ }^{3}$ Department of Biomedical Data Science, Geisel School of Medicine, Dartmouth College, 03756, Lebanon, USA. ${ }^{4}$ Annenberg School of Communications and Journalism, University of Southern California, Los Angeles, USA.

Received: 14 March 2019 Accepted: 23 July 2019

Published online: 23 September 2019

\section{References}

Al-Garadi MA, Varathan KD, Ravana SD, Ahmed E, Chang V (2016) Identifying the influential spreaders in multilayer interactions of online social networks. J Intell Fuzzy Syst 31(5):2721-2735

Azimi-Tafreshi N (2016) Cooperative epidemics on multiplex networks. Phys Rev E 93(4):042303

Bhaskaran K, Gasparrini A, Hajat S, Smeeth L, Armstrong B (2013) Time series regression studies in environmental epidemiology. Int J Epidemiol 42(4):1187-1195

Buldyrev SV, Parshani R, Paul G, Stanley HE, Havlin S (2010) Catastrophic cascade of failures in interdependent networks. Nature 464(7291):1025

Buono C, Alvarez-Zuzek LG, Macri PA, Braunstein LA (2014) Epidemics in partially overlapped multiplex networks. PloS ONE 9(3):92200

Cai W, Chen L, Ghanbarnejad F, Grassberger P (2015) Avalanche outbreaks emerging in cooperative contagions. Nat Phys 11(11):936

Centola D (2018) How Behavior Spreads: The Science of Complex Contagions vol. 3. Princeton University Press, Princeton Chang H, Fu F (2018) Co-diffusion of social contagions. N J Phys 20(9):095001

Czaplicka A, Toral R, San Miguel M (2016) Competition of simple and complex adoption on interdependent networks. Phys Rev E 94(6):062301

Erdős P, Rényi A (1960) On the evolution of random graphs. Publ Math Inst Hung Acad Sci 5(1):17-60

Foucquier J, Guedj M (2015) Analysis of drug combinations: current methodological landscape. Pharmacol Res Perspect 3(3)

Gomez S, Diaz-Guilera A, Gomez-Gardenes J, Perez-Vicente CJ, Moreno Y, Arenas A (2013) Diffusion dynamics on multiplex networks. Phys Rev Lett 110(2):028701

Grassberger P, Chen L, Ghanbarnejad F, Cai W (2016) Phase transitions in cooperative coinfections: Simulation results for networks and lattices. Phys Rev E 93(4):042316

Gu C-G, Zou S-R, Xu X-L, Qu Y-Q, Jiang Y-M, Liu H-K, Zhou T, et al (2011) Onset of cooperation between layered networks. Phys Rev E 84(2):026101

Guilbeault D, Becker J, Centola D (2018) Complex contagions: A decade in review. In: Lehmann S, Ahn Y-Y (eds). Complex Spreading Phenomena in Social Systems vol. 1. Springer, Cham. pp 3-25

Hagberg A, Schult D, Swart P, Conway D, Séguin-Charbonneau L, Ellison C, Edwards B, Torrents J (2013). Networkx. URL http://networkx.github.io/index.html

Hébert-Dufresne L, Althouse BM (2015) Complex dynamics of synergistic coinfections on realistically clustered networks. Proc Natl Acad Sci 112(33):10551-10556

Holme P, Kim BJ (2002) Growing scale-free networks with tunable clustering. Phys Rev E 65(2):026107

Jankowski J, Bródka P. Hamari J (2016) A picture is worth a thousand words: an empirical study on the influence of content visibility on diffusion processes within a virtual world. Behav Inf Technol 35(11):926-945

Jiang C, Chen Y, Liu KR (2014) Graphical evolutionary game for information diffusion over social networks. IEEE J Sel Top Signal Process 8(4):524-536

Liu Q-H, Zhong L-F, Wang W, Zhou T, Eugene Stanley H (2018) Interactive social contagions and co-infections on complex networks. Chaos: Interdiscip J Nonlinear Sci 28(1):013120

Liu Q-H, Wang W, Cai S-M, Tang M, Lai Y-C (2018) Synergistic interactions promote behavior spreading and alter phase transitions on multiplex networks. Phys Rev E 97(2):022311

Nowak MA, May RM (1994) Superinfection and the evolution of parasite virulence. Proc Royal Soc Lond B: Biol Sci 255(1342):81-89

Perc M, Marhl M (2006) Evolutionary and dynamical coherence resonances in the pair approximated prisoner's dilemma game. N J Phys 8(8):142

Perc M, Gómez-Gardeñes J, Szolnoki A, Floría LM, Moreno Y (2013) Evolutionary dynamics of group interactions on structured populations: a review. J Royal Soc Interf 10(80):20120997

Pérez-Reche FJ, Ludlam JJ, Taraskin SN, Gilligan CA (2011) Synergy in spreading processes: from exploitative to explorative foraging strategies. Phys Rev Lett 106(21):218701

Qiu W, Wang Y, Yu J (2012) A game theoretical model of information dissemination in social network. In: Complex Systems (ICCS), 2012 International Conference On. IEEE, Piscataway. pp 1-6

Shu P, Gao L, Zhao P, Wang W, Stanley HE (2017) Social contagions on interdependent lattice networks. Sci Rep 7:44669 
Srivastava A, Chelmis C, Prasanna VK (2016) Computing competing cascades on signed networks. Soc Netw Anal Min $6(1): 82$

Steger A, Wormald NC (1999) Generating random regular graphs quickly. Comb Probab Comput 8(4):377-396

Stella M, Selakovic S, Antonioni A, Andreazzi CS (2018) Ecological multiplex interactions determine the role of species for parasite spread amplification. eLife 7:32814

Szolnoki A, Perc M (2008) Coevolution of teaching activity promotes cooperation. N J Phys 10(4):043036

Szolnoki A, Perc M (2013) Information sharing promotes prosocial behaviour. N J Phys 15(5):053010

Szolnoki A, Perc M (2016) Collective influence in evolutionary social dilemmas. EPL (Europhysics Letters) 113(5):58004

Wang Z, Szolnoki A, Perc M (2014) Self-organization towards optimally interdependent networks by means of coevolution. N J Phys 16(3):033041

Wang Z, Wang L, Szolnoki A, Perc M (2015) Evolutionary games on multilayer networks: a colloquium. Euro Phys J B 88(5):124

Wang W, Liu Q-H, Liang J, Hu Y, Zhou T (2019) Coevolution spreading in complex networks. arXiv preprint arXiv:1901.02125

Watts DJ, Strogatz SH (1998) Collective dynamics of 'small-world'networks. Nature 393(6684):440

Zarazade A, Khodadadi A, Farajtabar M, Rabiee HR, Zha H (2017) Correlated cascades: Compete or cooperate. In: Thirty-First AAAI Conference on Artificial Intelligence. AAAI, Menlo Park

Zinoviev D, Duong V (2011) A game theoretical approach to broadcast information diffusion in social networks. In: Proceedings of the 44th Annual Simulation Symposium. Society for Computer Simulation International. pp 47-52

Publisher's Note

Springer Nature remains neutral with regard to jurisdictional claims in published maps and institutional affiliations.

\section{Submit your manuscript to a SpringerOpen ${ }^{\circ}$} journal and benefit from:

- Convenient online submission

- Rigorous peer review

- Open access: articles freely available online

- High visibility within the field

- Retaining the copyright to your article

Submit your next manuscript at $\boldsymbol{\triangleright}$ springeropen.com 